\title{
THE NEXT GENERATION OF PHOTON BEAM POSITION MONITORS FOR UNDULATOR BEAMLINES
}

\author{
A. Galimberti*, R. Borghes, G. Paolucci, R. Presacco SINCROTRONE TRIESTE \\ G. Paolicelli, INFM Unita' Roma Tre \\ G. Stefani, Universita' di Roma Tre and INFM Unita’ Roma Tre
}

\section{Abstract}

The detection of the real photon beam trajectory as delivered to the users, by a third generation SR facility, is of paramount importance for the effectiveness of the most sensitive experimental measurements. Photon Beam Position Monitors (PBPM) have been developed for this purpose. The most common is the so-called blade-typemonitor which is based on the photoemission principle [1, 2]. Unfortunately when it is used into an undulator beamline, it suffers from a background contamination due to the dipole radiation. So, a more complex device has been successfully developed at Elettra for counteracting this effect. The novel detector is based on spectroscopic techniques and collects signals essentially due to the undulator radiation only. In fact the residual error due to the dipoles is less than $0.1 \%$. Moreover it is easily tuneable on any desired working energy. The commissioning measurements have been carried on at ID4.2 and ID7.2 beamlines with undulator fundamental energies varying from few eV to $1 \mathrm{keV}$. A complete report about the results is presented on the present paper while the future perspectives of this detector are also fully discussed.

\section{INTRODUCTION}

A conventional diagnostic system, commonly used in storage rings, does not assure enough sensitivity to monitor the real photon beam delivered to the users.

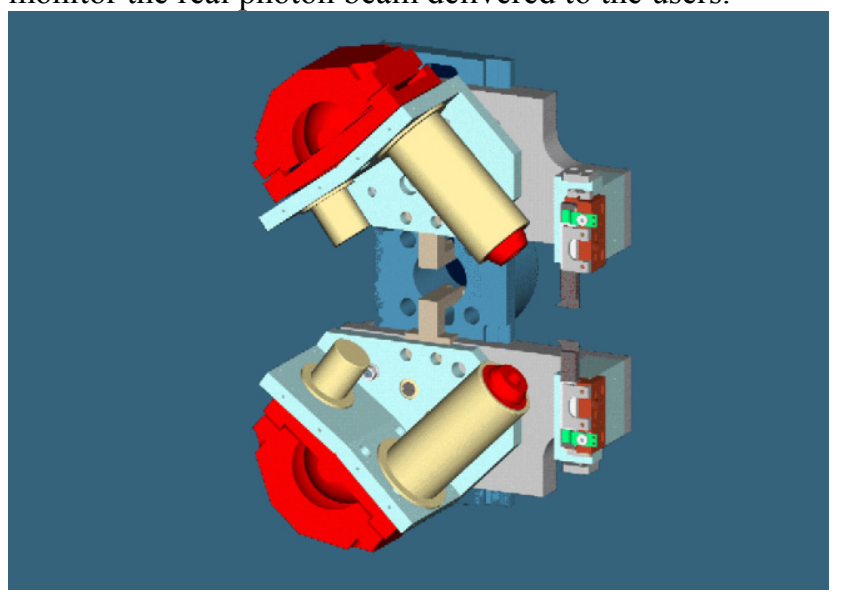

Figure 1: The NewPBPM prototype layout.

Operational experience, using the traditional photon beam position monitors, figures out how could be important any data collected by a detector insensitive to the dipole radiation. In fact, the photon beam delivered is affected by various unwanted effects like residual

\footnotetext{
galimberti@elettra.trieste.it
}

oscillations $(50 \mathrm{~Hz}$ and its harmonics, LFOs), drifts (thermal effects), jumps (orbit corrections, ID movements), spikes (magnet power supplies) which decrease the beam quality delivered to the experimental user. Some of them are dumped by orbit feedback, according with the eBPM data. But high sensitive experiments need corrections of angular effects down the micro-radian level. This level might be provided by PBPM only. Unfortunately, for beamlines fed by undulators and highly sensitive to beam instabilities, it is not available a reliable PBPM. Various attempts have been made to overcome this limitation [3, 4] but no definitive solutions have been proposed yet. At Elettra, a not conventional approach has been taken and a novel detector has been developed, called New Photon Beam Position Monitor (NPBPM) [5]. It is based on spectroscopic techniques and collects, from a common set of blades impinging the beam, the photo-emitted electrons. A further selection, over their energies, permits to get a signal essentially due only to photons with an energy included in a narrow bandwidth, peaked on the desired undulator emission. This treatment allows a drastic reduction of the dipole radiation contamination from the signals used to compute the photon beam centre and its trajectory.

\section{THE DETECTOR}

The two main components of the NPBPM are: a conventional set of two galvanic insulated blades coupled to a pair of electron energy selectors.

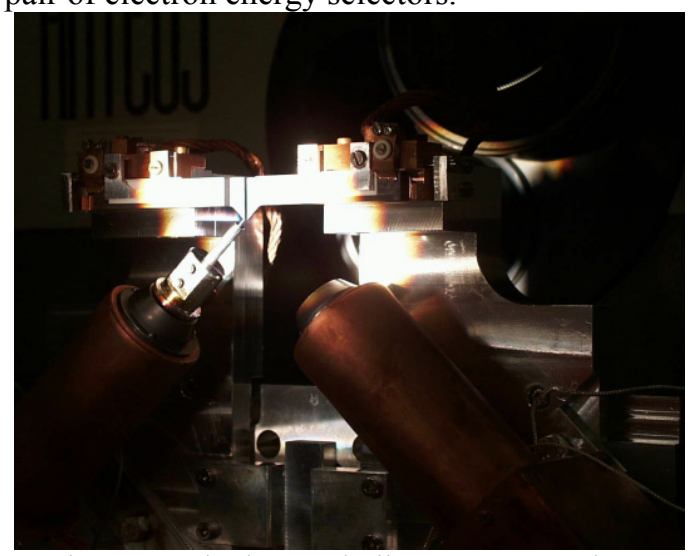

Figure 2: The internal alignment procedure.

The effectiveness of the system depends on the electron energy selector adopted. It has to be designed and optimised for the energy range of each undulator source. Besides, its energy bandwidth influences the maximum residual error due to the dipoles. A first prototype has 
been developed and its layout is depicted in fig. 1. It detects the vertical position only and it adopts two hemispherical dispersing elements with three electrostatic input lenses. The mutual alignment blade-analyser has very strict tolerances for the low angular acceptance of the electrostatic device itself which is less than a tenth of mradiants (fig.2). The particular geometric layout of the detector provides output data without crosstalk error. Tuning the analysers at the proper energy, depending on undulator spectrum and blade photoemission line, the NPBPM rejects almost all the dipole radiation contributions to the signal.

\section{DETECTOR HIGHLIGHTS}

\subsection{Dipole Radiation Contamination}

The analysers have a pass energy of some tenths of $\mathrm{eV}$. Tuning the detector on the proper energy peak due to the undulator, the maximum residual error due to the dipole radiation contamination is dumped below $0.1 \%$. The photons emitted by the undulator shows their predicted bigaussian behaviour (fig.3). No contamination due to other sources appear superimposed over its shape.

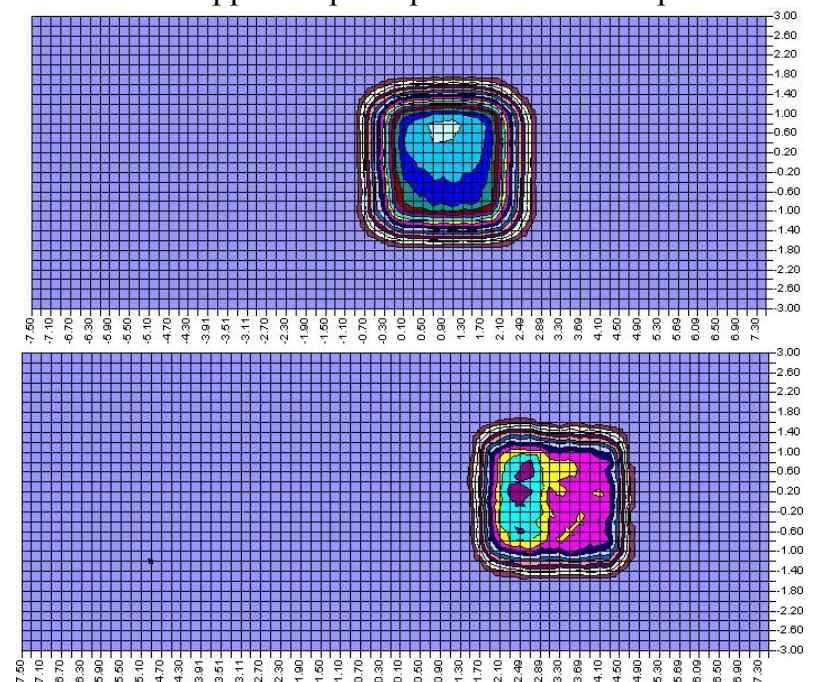

Figure 3: The undulator photon beam seen by the two electron energy analysers performing a spatial scan.

This condition allows operations virtually free from dipole error. In figure 4 is clearly depicted the dipole contamination effect both on the traditional detector (PBPM) and on the novel device (New PBPM) [6]. The NPBPM selectivity over the photon energies has an impressive effect over the integrated signal collected. Moreover the NPBPM signal is entirely due to a wellknown restricted area of the photoemissive blades located close at their edge. So, the comissioning data confirms that when we select signals due to a narrow energy bandwidth around an undulator harmonic only, we decrease the BM contamination of about 100 times. This effect is due to the predicted flux difference between the $\mathrm{BM}$ and the undulator in the energy range considered. In fact the drastic reduction of the energy and spatial integration windows allows to really shrink down the dipole with respect to the undulator radiation effect on the NPBPM outputs.
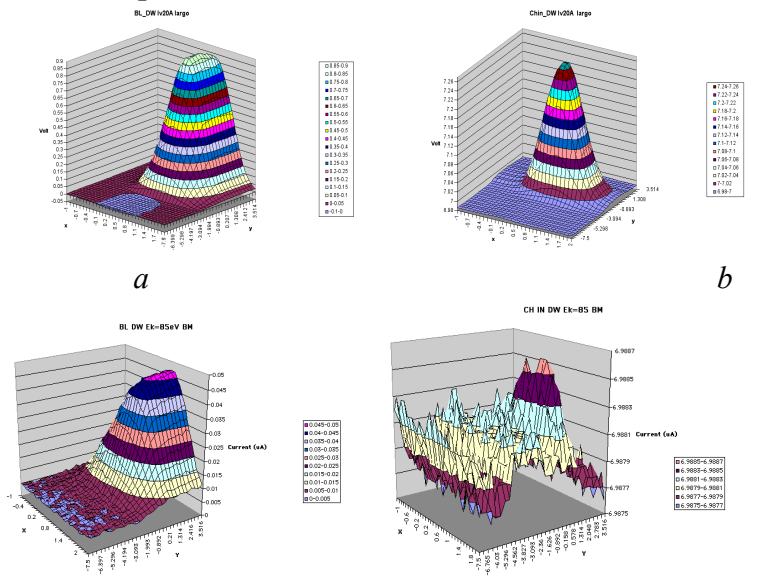

c

Fig. 4: A spatial scan shows the photon beam as seen by the different electrodes: a) Blade PBPM b) New PBPM c)Blade PBPM (only dipole) d) New PBPM (only dipole) in an Undulator beamline.

\subsection{Photon Energy Range}

The first detector developed is optimised for an energy range from few $\mathrm{eV}$ to $1 \mathrm{keV}$. Anyway, there are no technical problem to design further models suitable to work at higher energies. The NPBPM is actually equipped with a graphite set of blades. This material forces to work with only two choices: the valence band and the carbon 1s electron level. For higher undulator energies it might be better to use heavier materials, like $\mathrm{Au}$. In fact, more electron energetic levels might help the detection of photo-electrons of higher energies.

\subsection{Linearity Range and Sensitivity}

The NPBPM, performing a sort of bandpass filtering and having a restricted field of view, rejects the majority of the photo-electrons emitted by the blades.

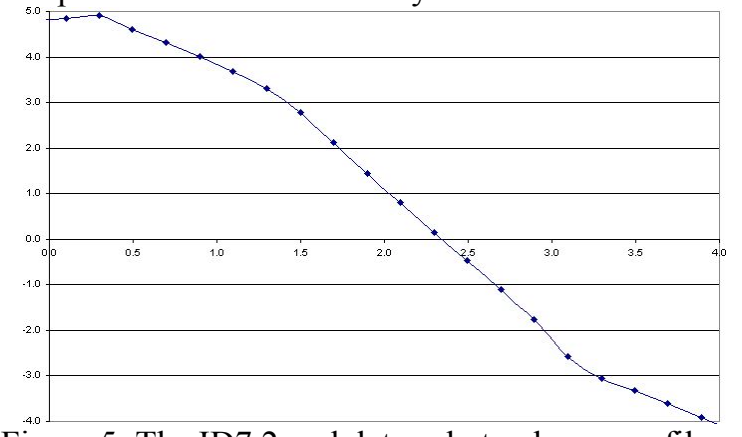

Figure 5: The ID7.2 undulator photon beam profile as seen by the NPBPM (fundamental peak @ 145eV).

The effect is a drastic reduction of the signal level available. Using electron multipliers, due to their not predictable efficiency over the time, may cause maintenance troubles. So, we have adopted simple 
Faraday cups coupled to a custom high sensitive electronics. Another effect that we have taken in account is the strong dependency of the NPBPM sensitivity over the undulator harmonic behaviour. Infact, the detector has different linear ranges and sensitivities for each undulator harmonic considered. In fig. 5 is shown the beam profile, of the ID7.2 with a gap of $50 \mathrm{~mm}$. The linearity range is about $1.5 \mathrm{~mm}$ wide and the sensitivity is of $3 \mathrm{pA} / \mu \mathrm{m}$. The slope of the curve changes according to the undulator settings applied.

\section{INSTRUMENTATION}

Each electrostatic element needs a HV floating power supply channel with a very low voltage ripple. So, we can refer one voltage to another and tune the NPBPM at the proper energy without significant fluctuations on its value. The intrinsic stability of any applied voltage, is assured by a HV power supply system, manufactured by C.A.E.N. and Elettra, developed for the high resolution electron analysers used in experimental physics. The data acquisition, at the analyser exit, is done in current mode and it is supported by electron multipliers or by simple Faraday cups. The latter have a very low signal level (i.e. some pA for $1 \mathrm{um}$ ). So, at Elettra, has been developed a modular smart instrumentation, called Picolite. It essentially is a double channel floating transimpedence amplifier fully handled by embedded microcontrollers. This instrument allows the detection of currents as low as $0.1 \mathrm{pA}$ and as high as $1 \mathrm{~mA}$. It is fully controllable by serial link or by some digital I/O bits. It provides an on board 16 bit A/D conversion performed at the bias voltage applied to the analyser exit. A smart software procedure tunes the NPBPM on the desired undulator configuration. It verifies the undulator harmonic energy and it moves the gap between the blades according to the beam profile of the harmonic considered.

\section{OPERATIONAL EXPERIENCE}

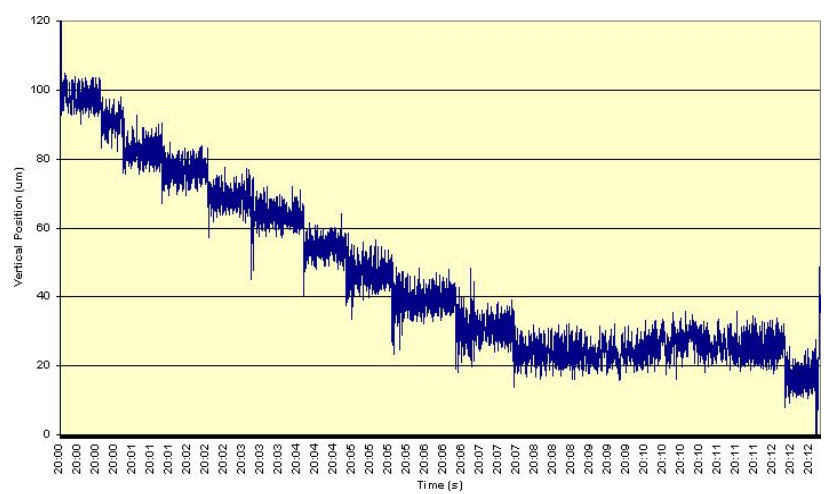

Figure 6: Real undulator photon beam position monitoring, at the ID7.2 beamline, using the NPBPM. The detector has been tested on two different beamlines: ID4.2 and ID7.2. The photon fundamental energy was from few $\mathrm{eV}$ to $500 \mathrm{eV}$. It was installed at about $15 \mathrm{~m}$ far from the source. The NPBPM has a real micron level sensitivity and the photon beam monitoring, shown in figure 6 , follows the real undulator beam movements. Using two NPBPM, $10 \mathrm{~m}$ far each other, an angular sub-microradian sensitivity may be obtained. Moreover, an auto-tuning procedure adjusts the detector parameters, following the photon beam changing versus the ID gap movements.

\section{CONCLUSIONS}

The detector proposed has fully obtained its goals: to dump the dipole radiation contamination and to have a micron sensitivity. At Elettra a first production of the promising device has started and first results on the ultrahigh precision local beam position stabilisation, on one of the most sensitive beamline, are foreseen for the next year. At present a couple of detectors is under design for upgrading the traditional PBPM system installed on the SuperESCA beamline fed by the ID 2.2 undulator source. These devices will be more compact than the prototype and will be active on both horizontal and vertical planes. A complete re-engineering of the device is undergoing and it will allow also a drastic cut off of its cost. Besides, a study has started for the design of another detector optimised for energies up $2 \mathrm{keV}$. The main target to stabilise the photon beam, seen by the user, seems now really possible also for the most demanding undulator beamlines.

\section{ACKNOWLEDGEMENTS}

The authors would like to thank J. Grgic, C. Fava and A. Gambitta for their technical skill and friendly collaboration. We are also in debt with the scientific staff of the Aloisa beamline, co-ordinated by A. Morgante.

\section{REFERENCES}

[1] E.D. Johnson and T. Oversluizen, Rev. Sci. Instrum. 60 (7), July 1989.

[2] A. Galimberti et al.: 'Photon Beam Position Monitors suitable for a Local Feedback System at ELETTRA', Proc. 5th EPAC, Sitges, Spain, 1996.

[3] G. Decker and O. Singh: 'Method for Reducing XRay Background Signals from Insertion Device XRay Beam Position Monitors', PRST-AB, Vol.2, 112801, 1999.

[4] A. Galimberti and C. Scafuri: 'A Preliminary Study on High Precision Photon Beam Position Monitor Design for Local Feedback Systems', Proc. 6th EPAC, Stockholm, Sweden, 1998

[5] A. Galimberti et al.: 'A New Approach to Photon Beam Position Monitoring at Elettra', Proc. 18th PAC, New York, NY, USA, 1999.

[6] A. Galimberti et al.: 'First Results of the Novel Photon Beam Position Monitor for Undulator Beamlines of Elettra', Proc. SRI 2000, Berlin, Germany, 2000 (to be published on NIMA). 